\title{
Anti-apoptotic effect of retinoic acid on retinal progenitor cells mediated by a protein kinase A-dependent mechanism
}

\author{
Roman Kholodenko ${ }^{1}$, Irina Kholodenko ${ }^{1}$, Viktor Sorokin ${ }^{2}$, Anna Tolmazova ${ }^{1}$, Olga Sazonova ${ }^{1}$, Anton Buzdin ${ }^{1}$ \\ ${ }^{1}$ Shemyakin-Ovchinnikov Institute of Bioorganic Chemistry, 16/10 Miklukho-Maklaya, Moscow 117997, Russia $;{ }^{2}$ Russian State Medi- \\ cal University, Ostrovityanova 1, Moscow 117312, Russia
}

Retinal progenitor cells (RPCs) are neural stem cells able to differentiate into any normal adult retinal cell type, except for pigment epithelial cells. Retinoic acid (RA) is a powerful growth/differentiation factor that generally causes growth inhibition, differentiation and/or apoptosis. In this study, we demonstrate that RA not only affects mouse RPC differentiation but also improves cell survival by reducing spontaneous apoptotic rate without affecting RPC proliferation. The enhanced cell survival was accompanied by a significant upregulation of the expression of protein kinase A (PKA) and several protein kinase C (PKC) isoforms. Treatment of cells grown in RA-free media with 8-bromoadenosine 3',5'-cyclic monophosphate, a known activator of PKA, resulted in an anti-apoptotic effect similar to that caused by RA; whereas the PKA inhibitor N-[2-(p-bromocinnamylamino)ethyl]-5-isoquinolinesulfonamide dihydrochloride led to a significant $(\sim 32 \%)$ increase in apoptosis. In contrast, treatment of RPCs with any of two PKC selective inhibitors, 2,2',3,3',4,4'-hexahydroxy-1,1'-biphenyl-6,6'-dimethanol dimethyl ether and bisindolylmaleimide XI, led to diminished apoptosis; while a PKC activator, phorbol 12-myristate 13-acetate, increased apoptosis. These and other data suggest that the effect of RA on RPC survival is mostly due to the increased anti-apoptotic activity elicited by PKA, which might in turn be antagonized by PKC. Such a mechanism is a new example of tight regulation of important biological processes triggered by RA. Although the detailed mechanisms remain to be elucidated, we provide evidence that the pro-survival effect of RA on RPCs is not mediated by changed expression of p53 or bcl-2, and appears to be independent of $\beta$-amyloid, Fas ligand, TNF- $\alpha$, ganglioside GM1 and ceramide C16-induced apoptotic pathways.

Cell Research (2007) 17:151-162. doi: 10.1038/sj.cr.7310147; published online 13 February 2007

Keywords: retinal progenitor cells, apoptosis, retinoic acid, protein kinase A, protein kinase C

\section{Introduction}

In mammals, retinogenesis mostly proceeds during embryo development and is completed within a few days after

\footnotetext{
Correspondence: Anton Buzdin

Tel: +7-495-330-6574; Fax: +7-495-330-6538

E-mail: anton@humgen.siobc.ras.ru

Received 12 May 2006; revised 25 September, 28 November 2006; accepted 19 December 2006; published online 13 February 2007

Abbreviations: retinal progenitor cells (RPC); all-trans-retinoic acid (RA); protein kinase C (PKC); protein kinase A (PKA); N-(2-[p-bromocinnam ylamino]ethyl)-5-isoquinolinesulfonamide dihydrochloride (H89); 8-bromoadenosine $3^{\prime}, 5^{\prime}$-cyclic monophosphate (8-Br-cAMP); 2, 2',3,3',4,4'-hexahydroxy-1,1'-biphenyl-6,6'-dimethanol dimethyl ether (HBDDE); bisindolylmaleimide XI (BIM); phorbol 12-myristate 13-acetate (PMA)
}

birth. Retinal stem cells have the potential to produce either neural retinal progenitor cells (RPCs) or retinal pigment epithelial progenitors. RPCs generate seven known retinal neuronal cell types: rod and cone photoreceptor cells, required for black-white and color visions, respectively; Müller glia cells; bipolar, ganglion, horizontal and amacrine neurons, which perceive signals from photoreceptor cells, transform them and transduce them to the brain [1]. Although neuronal stem cell niches were found in brains of several mammalian species $[2,3]$, adult organisms are thought to lack any kind of neuronal retinal cell population renewal $[4,5]$. Therefore, the treatment of various retinal disorders would require the use of neuronal stem cells to replace damaged retinal neurons or glia [6]. Indeed, in 
animal models the approach of stem cell therapy was rather efficient for the treatment of diverse neurodegenerative diseases, such as Huntington's and Parkinson's diseases [7-9], as well as for curing some retinal disorders [1012]. RPCs would be useful for retinal disease treatment because of their natural ability to differentiate into any of the above-mentioned retinal neuronal cell classes. Several inheritable and acquired retinopathies, including Retinitis Pigmentosa, are associated with the loss of particular retinal cell types [13].

It was shown that RPCs can be isolated from pre-natal or few days post-natal mouse [14], rat [15] and human $[16,17]$ retinas and can be cultured in vitro. Although there is a great difference between in vivo and in vitro stem cell differentiation and survival [18], the knowledge obtained from in vitro experiments will be necessary for the development of new in vivo assays and new therapeutic strategies. Ezeonu et al. [19] demonstrated that cell commitment in human retinal precursor cells could be modified in response to exogenous growth factors such as basic fibroblast growth factor and transforming growth factor- $\alpha$. Moreover, RPC differentiation was density dependent: a larger proportion of multipotential precursors plated at a density of $1 \times 10^{4}$ cells $/ \mathrm{cm}^{2}$ differentiated into cells displaying photoreceptor phenotypes as compared with cells plated at higher densities, and the effects were enhanced in response to growth factors. Kelley et al. [20] used two photoreceptor-specific antibody markers, anti-recoverin (expressed in all photoreceptors) and anti-rhodopsin (specific for rod photoreceptors) to assess the effects of retinoic acid (RA) on differentiation of photoreceptor phenotypes in vitro. They found that RA treatment led to an increase in the number of cells expressing these markers after 2-8 days. This effect of RA on photoreceptor differentiation was specific, as the other major cell type produced at this stage in development, the amacrine cells, did not increase with RA treatment, but instead their productions were inhibited [21]. Recently, we have established a primary mouse RPC cell culture system and investigated the effects of various growth/differentiation factors on RPC phenotype and differentiation, including transforming growth factor- $\alpha$, basic fibroblast growth factor, nerve growth factor, epidermal growth factor, platelet-derived growth factor, neurotrophin 3 and RA (unpublished data). The only factor found to reproducibly alter RPC phenotype was RA, while the others did not display any significant effects on RPC survival, morphology and marker protein expression, which might be due to the presence of these factors in the fetal serum used in culture media. RA-treated RPCs showed improved viability and decreased immunoreactivity to antibodies against nestin, a neuroepithelial stem cell marker protein, whereas expression of the proliferative marker $\mathrm{Ki}$ 67 remained unchanged.

RA, known to be a powerful regulator of cell fate, regulates many aspects of development. In the developing retina, RA is produced at high concentrations, and several lines of evidence implicate a role for this molecule in photoreceptor development [21]. However, our observation that RA treatment leads to improved RPC viability represents a new finding, and the present study is focused on examining the underlying mechanisms. We report here that the effect of RA is due exclusively to a decrease in the spontaneous apoptotic rate of RPCs, through a mechanism most probably mediated by increase expression of protein kinase A (PKA), whose anti-apoptotic activity is in turn negatively modulated by protein kinase $\mathrm{C}$ (PKC).

\section{Materials and Methods}

\section{Animals}

All the experiments with mice were performed in accordance with both the Russian and the European Communities Council Directive (86/609/EEC) and institutional guidelines, and followed the tenets of the Declaration of Helsinki. In all cases, efforts were made to minimize suffering of the animals. CBA strain mice were used throughout the study. The plug date of timed pregnant mice was defined as embryonic day 0 .

\section{RPC isolation}

Embryonic retinal tissues were isolated from day 14 mouse embryos. Eyes from 15 embryos were isolated and washed twice in $1 \times$ PBS standard buffer solution (ICN, USA) with added claforan (10 $\mu \mathrm{g} / \mathrm{ml})$ and lincomycin $(50 \mu \mathrm{g} / \mathrm{ml})$ antibiotics. Neural retinas were isolated by removing the retinal pigment epithelium and vitreous body. The retinas were then dissected and approximately $1 \mathrm{~mm}^{2}$ fragments were incubated in $0.25 \%$ trypsin solution (PanEko, Russia) in PBS buffer for $30 \mathrm{~min}$ at $37^{\circ} \mathrm{C}$ to homogenize the tissue. The trypsinization was then stopped by adding fetal calf serum (FCS) (HyClone, USA) to a concentration of $10 \%$. The cells were washed twice in PBS, transferred to DMEM/F12 medium (Sigma, USA) supplemented with $10 \% \mathrm{FCS}$, and plated in six-well polystyrene culture plates (Corning Costar, USA) at a density of $5 \times 10^{5}$ cells $/ \mathrm{cm}^{2}$. Approximately $90 \%$ of the cells were viable. For viable cell counts, an aliquot was counted by the trypan blue (Sigma, USA) exclusion assay in a hemocytometer.

\section{Media and culturing conditions}

Media for cell culturing were DMEM/F12 (Sigma, USA) $(0.2 \%$ $\mathrm{NaHCO}_{3}, \mathrm{pH}$ 7.4) with $10 \%$ FCS, 2 mM L-glutamine (ICN, USA) and $25 \mu \mathrm{g} / \mathrm{ml}$ linkomycin. Cultures were maintained at $37{ }^{\circ} \mathrm{C}$ in an incubator with $96 \%$ air and $4 \% \mathrm{CO}_{2}$. Cells were cultivated in multi-well plates or in cell culture flasks at densities of $5 \times 10^{5}$, $1 \times 10^{5}, 5 \times 10^{4}$ and $1 \times 10^{4}$ cells $/ \mathrm{cm}^{2}$. Cell passaging was performed every 2-6 days, depending on the cultured cell proliferation rate, at $75 \%$ confluence. Cells were harvested by double washing with trypsin/versen solution ( $0.25 \%$ trypsin, $0.02 \%$ EDTA) in PBS and then centrifuged at $300 \times g$. 


\section{Monitoring of RA effects}

To explore the effects of RA on cell survival and differentiation, RPCs of passage 8 were plated with different density on plastic culture dishes. For cell culture assays, DMSO-solubilized all-trans-RA (Sigma, USA) was added to cultured RPCs to a final concentration of $100 \mathrm{ng} / \mathrm{ml}$. The culture medium was changed and the growth factors replenished every 2 days. The cells were monitored by darkfield and phase-contrast microscopy over a period of 1-7 days, and finally (after 7-day incubation) analyzed in immunocytochemistry experiments.

\section{Light microscopy}

Cell cultures were examined using an inverted light microscope Axio Vert 200 (Carl Zeiss, Germany). Samples were photographed with a digital camera AxioCam HRm (Carl Zeiss, Germany) at 10$80 \times$ magnification. For imaging and data analysis, the KS400 (version 3.0) software was used.

\section{Immunocytochemistry}

Cells for immunocytochemical and flow cytometry analyses were harvested with trypsin/versen solution ( $0.25 \%$ trypsin), double washed with PBS and fixed for 30 min with phosphate-buffered $2 \%$ paraformaldehyde (PanEko, Russia) at $4{ }^{\circ} \mathrm{C}$. The fixed samples were washed twice in PBS and permeabilized in $0.6 \%$ saponin (Sigma, USA) PBS solution for $30 \mathrm{~min}$ at room temperature. The samples were then double washed with PBS containing $1 \%$ FCS and incubated for $1 \mathrm{~h}$ at $4{ }^{\circ} \mathrm{C}$ with the following primary anti-mouse antibodies (Santa Cruz, USA): anti-rhodopsin, mixed anti-green/blue opsins, anti-nestin, anti- $\beta$-arrestin, anti-calbindin, anti-calretinin, anti-recoverin, anti-Ki-67, anti-glial fibrillary acidic protein (GFAP) and anti-PKC $\alpha$. For RA anti-apoptotic activity studies, anti-bcl-2 and anti-p53 antibodies (Sigma, USA) were used. Each representative sample of $5 \times 10^{5}$ cells was incubated with $2 \mu \mathrm{g}$ of primary antibodies in $100 \mu \mathrm{l}$ of $1 \times \mathrm{PBS} / 10 \% \mathrm{FCS}$. The samples were washed twice with $1 \times \mathrm{PBS} / 1 \% \mathrm{FCS}$, incubated for $1 \mathrm{~h}$ with fluorescein isothiocyanate (FITC)-conjugated secondary antibodies (Santa Cruz, USA) at $4{ }^{\circ} \mathrm{C}$, and then washed and fixed in $2 \%$ paraformaldehyde solution, as described above. Immunoreactivity analyses with a minimum of 5000 events per sample were performed on an EPICS Coulter XL-MCL flow cytometer. For fluorescent microscopy, cells were grown on glass slides in eight-well culturing plates. Such a visualization was used here as a raw control for flow cytometry results.

Staining with antibodies was performed as described above except that phycoerythrin-conjugated secondary antibodies (Santa Cruz, USA) were used. Nuclear stains were performed using 4',6diamidino-2-phenylindole, dihydrochloride (DAPI; Molecular Probes, USA). After antibody staining, cell nuclei were labeled with DAPI $(0.1 \mu \mathrm{g} / \mathrm{ml}, 5-\mathrm{min}$ incubation) and washed twice with PBS. Samples were photographed with a digital camera AxioCam HRm (Carl Zeiss, Germany) at 10-80× magnification. For imaging and data analysis, the KS400 (version 3.0) software was used.

\section{Propidium iodide staining for apoptotic cells and cell cycle analyses}

Cell cycle/apoptosis analyses were performed using propidium iodide (PI) staining with subsequent FACS analysis. Prior to PI staining, cells were fixed in $70 \%$ ethanol $\left(1 \mathrm{~h}\right.$ incubation at $\left.4^{\circ} \mathrm{C}\right)$, and double washed by suspending in PBS with a subsequent 10-min centrifugation at $300 \times \mathrm{g}$. The cell pellets were further resuspended in DNA staining solution $(1 \times \mathrm{PBS}, 20 \mu \mathrm{g} / \mathrm{ml}$ propidium iodide (Merck), $50 \mu \mathrm{g} / \mathrm{ml}$ RNase A(Fermentas)). For all samples, cell death analysis was performed in triplicate. An EPICS Coulter XL-MCL flow cytometer was used to evaluate apoptotic cell numbers with a minimum of 2000 events analyzed per each sample. The data files were saved automatically by the CellQuest software (BD Biosciences), and WinMDI (version 2.8; The Scripps Institute, USA) was used to perform quadrant analysis. A two-tailed $t$-test to determine the significant difference between treatment and control experiments was performed using Excel (Microsoft). For cell cycle analyses, WinMDI and FCS Express programs were used.

\section{Annexin V assay for apoptotic cell measurements}

To confirm the results of PI staining for apoptotic cells, Annexin V assay kit (Molecular Probes) was used. RPCs were assayed by the detection of membrane externalization of phosphatidylserine with Annexin V-FITC conjugate. Briefly, $3 \times 10^{5}$ cells were harvested after 7-day RA treatment, washed twice with cold PBS and resuspended in $200 \mu \mathrm{l}$ of binding buffer. Both adherent and floating cells were harvested for the apoptosis assay. Annexin V-FITC and PI $(1 \mu \mathrm{g} / \mathrm{ml})$ were added to individual samples and incubated for $15 \mathrm{~min}$ in a dark environment. The reaction was stopped by adding $300 \mu \mathrm{l}$ of binding buffer. Then the cells were analyzed with EPICS Coulter XL-MCL flow cytometer.

\section{Cell growth/proliferation}

For cell growth and proliferation studies, both colorimetric MTT (3-[4,5-dimethylthiazol-2-yl]-2,5-diphenyltetrazolium bromide) and flow cytometric BrdU (bromo-2'-deoxyuridine) assays were performed. All MTT experiments were reproduced four times. An aliquot of the MTT solution (Sigma) was added to each sample for $4 \mathrm{~h}$. The optical density (OD) was read in a Multiscan microplate reader (Titertek, Switzerland) at a test wavelength of $540 \mathrm{~nm}$. Cell proliferation assay was based on BrdU incorporation into newly synthesized DNA of proliferating cells. Cells were plated in defined media supplied with $10 \%$ FCS containing 0-1 $000 \mathrm{ng} / \mathrm{ml} \mathrm{RA}$ for 7 days. During the last hour of incubation, BrdU was added to the cultures at $10 \mu \mathrm{M}$. The solution was then removed and the cells were stained with FITC-coated anti BrdU antibodies according to a standard protocol [22].

\section{Inhibitor/activator assay}

The following protein kinase effector molecules were used: PKA inhibitor H89 ( $N$-[2-( $p$-bromocinnamylamino)ethyl]-5-isoquinolinesulfonamide dihydrochloride), $10 \mu \mathrm{M}$ (Sigma); PKA activator 8-Br-cAMP (8-bromoadenosine 3',5'-cyclic monophosphate), $10 \mu \mathrm{M}$ (Sigma); PKC inhibitors HBDDE (2,2',3,3',4,4'-hexahydroxy-1,1'biphenyl-6,6'-dimethanol dimethyl ether), 5-10 $\mu \mathrm{M}$ (Calbiochem), and BIM (bisindolylmaleimide XI), $1 \mu \mathrm{M}$ (Sigma); PKC activator PMA (phorbol 12-myristate 13-acetate), $10 \mu \mathrm{M}$ (Sigma). They were added separately or in combination to RPC cultures for a period of 7 days, and apoptotic cells were then analyzed by PI staining (see above).

\section{Induction of apoptosis}

RPCs were incubated for 3 days with the following known inducers of apoptosis in neuronal cells: $\beta$-amyloid $(50 \mu \mathrm{M})$, ceramide $\mathrm{C} 16$ $(20 \mu \mathrm{M})$, anti-Fas antibodies $(1 \mu \mathrm{g} / \mathrm{ml}), \mathrm{TNF}-\alpha(1 \mu \mathrm{g} / \mathrm{ml})$ and GM1 ganglioside $(40 \mu \mathrm{g} / \mathrm{ml})$. 

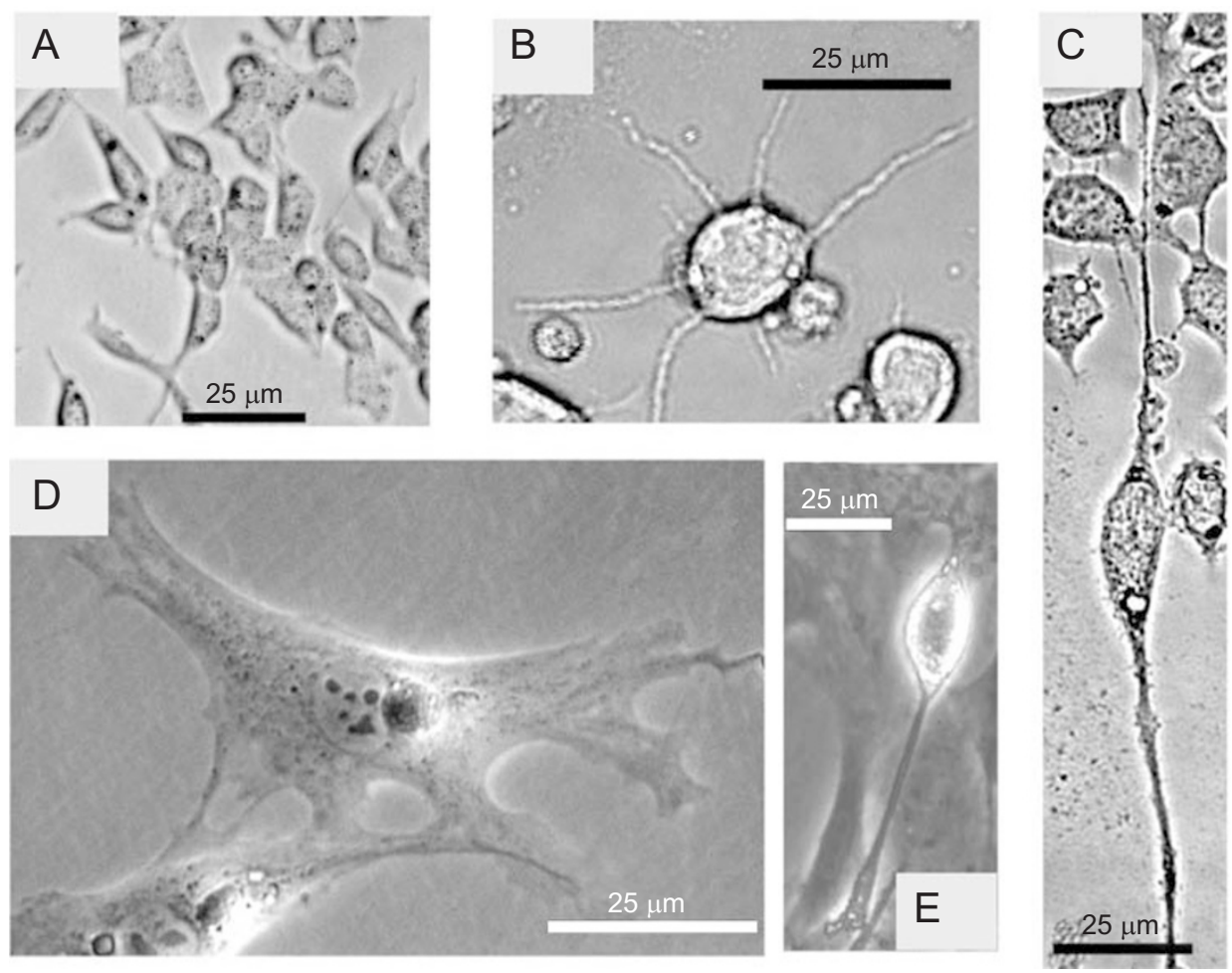

Figure 1 Phase-contrast photographs showing the morphologies of differentiated and undifferentiated RPCs. Magnification bar: $25 \mu \mathrm{m}$. (A) Undifferentiated RPCs, 7 days after plating at a cell density of $5 \times 10^{5} \mathrm{cells} / \mathrm{cm}^{2}$. (B) Differentiated RPCs displaying ganglion cell-like morphology. (C) Differentiated RPCs displaying bipolar neuron-like morphology. (D) Differentiated RPCs similar to astrocyte and Müller glia cells. (E) Photoreceptor-like morphologies of the differentiated RPCs.

\section{DNA sequence analysis}

Mouse genomic sequences and sequences of mouse gene transcripts were accessed through the UCSC genome browser (http://genome.ucsc.edu/cgi-bin/hgBLAT).

\section{Oligonucleotides}

Primers were synthesized using an ASM-102U DNA synthesizer (Biosan, Novosibirsk, Russia). The following oligonucleotides were used: PKC_A_for, 5'-gga ctt atc cac caa ggg atg-3' and PKC_A_rev, $5^{\prime}$-cca ggt tgt tgg atg gtt $\mathrm{g}-3^{\prime}$ (for PKC $\alpha$ isoform expression); PKC_E_for, 5'-ctg gtc get gcg cca tgc-3' and PKC_E_rev, 5'-atg ttg acg ctg aac cgt tg-3' (for PKC $\varepsilon$ isoform expression); PKC_Z_for, $5^{\prime}$-tac agg tgc atc aac tgc aag-3' and PKC_Z_rev, 5'-cgt cgg cat caa gga gga-3' (for PKC $\zeta$ isoform expression); PKC_Q_for, 5'-gaa aca acc gtg gaa ctc tac tc-3' and PKC_Q_rev, $5^{\prime}$-agt cct tgc ctc gec agc-3' (for PKC $\theta$ isoform expression); PKC_B_for, $5^{\prime}$-aag acc atc aag tgt tcc ctc a-3' and PKC_B_rev, $5^{\prime}$-tgc act cca cat cgt cat ctt-3' (for PKC $\beta-1$ isoform expression); PKC_G_for, $5^{\prime}$-cga ttt aat gta cca cat cca gc-3' and PKC_G_rev, $5^{\prime}$-acc tct ccc aat cga tcc aac-3' (for PKC $\gamma$ isoform expression); PKC_D_for, $5^{\prime}$-gcc tca aca agc aag gct aca-3' and PKC_D_rev, 5'-cag tcc gca gat gat ctc agc-3' (for PKC $\delta$ isoform expression); PKC_I_for, $5^{\prime}$-gat ccg cat ccc gcg ttc-3' and PKC_I_rev, 5'-cac tct cga atc ctg cet ctg a-3' (for PKC $\lambda / \mathrm{i}$ isoform expression); $\mathrm{PKC} \_\mathrm{H} \_$for, $5^{\prime}$-gaa gac gag tcc atc aag tga ac-3' and PKC_H_rev, $5^{\prime}$-gcc gaa tct gct gga aga g- $3^{\prime}$ (for PKC $\beta$ isoform expression); PKA_A_for, 5'-tcc tgg ggg aca ccc tgg gcg-3' and PKA_A_rev, 5'-ggc ttc gtt gtg ttg agt gca tcc-3' (for PKA $\alpha$ catalytic subunit expression); PKA_B_for, $5^{\prime}$-caa gag gct tgc aaa gac atc-3' and PKA_B_rev, $5^{\prime}-\mathrm{ccg}$ ttc tct tca act tct gat tta c-3' (for PKA $\beta$ regulatory subunit expression).

\section{RNA isolation and cDNA synthesis}

Total RNA was isolated from frozen cells pulverized in liquid nitrogen using an RNeasy Mini RNA purification kit (Qiagen). All RNA samples were further treated with DNase I to remove residual DNA. Full-length cDNA samples were obtained according to a cap switch effect-based SMART cDNA synthesis protocol (Clontech, BD Biosciences) using an oligo (dT)-containing primer and PowerScript reverse transcriptase (Clontech, BD Biosciences).

\section{$R T-P C R$}

All RT-PCR experiments described in this section were reproduced at least three times using independent cDNA preparations. For RT-PCR control of cDNA quality, we used two primer sets specific for the $\beta$-actin gene transcript $5^{\prime}$ and $3^{\prime}$ parts, respectively (Gene Checker Kit, Invitrogen). To find out transcription levels of selected genes in RPC samples under study, we performed a series of RT-PCR experiments with primers predominantly designed against neighboring constitutive exons in the middle part of the corresponding cDNA molecule. Prior to the RT-PCR analysis, the priming efficiency of the 
primers was pre-examined by genomic PCRs at temperatures varying depending on the primer combination used. These PCRs were carried out for 19, 22, 25 and 28 cycles, with $40 \mathrm{ng}$ of the mouse genomic DNA isolated from RPCs using a Wizard SV Genomic DNA Purification System (Promega). The RT-PCR was performed with cDNA samples obtained from RA-treated and untreated RPCs, an equivalent of $20 \mathrm{ng}$ total RNA being used as template in each PCR reaction performed in a final volume of $40 \mu \mathrm{l}$. Aliquots $(5 \mu \mathrm{l})$ of the reaction mixture after $21,24,27,30,33,36$ and 39 cycles of the amplification were analyzed by electrophoresis in $1.5 \%$ agarose gels. In all cases, the transcriptional status was determined from the number of PCR cycles needed to detect a PCR product of the expected length, and the PCR product concentration was measured using a Photomat system and the Gel Pro Analyzer software.

\section{Results}

Cultured RPCs display characteristic morphologies and express marker proteins of retinal neurons

Multipotential RPCs were isolated from day 14 mouse embryos, as the differentiation of retinal precursors has not yet been completed at this time point [1]. To characterize the primary cell culture, we investigated its ability to display typical retinal cell phenotypes, either by expressing marker proteins or by demonstrating characteristic cell shapes. In line with previous findings [19], the major RPC differentiation factor was the cell density: more than $70 \%$ of the cells plated at the densities of $1 \times 10^{4}$ or $5 \times 10^{4} / \mathrm{cm}^{2}$ differentiated into various retinal neuron-like cell types, in contrast to cells plated at high density which tended to retain their undifferentiated phenotype (Figure 1A). Another fac- tor was the concentration of FCS in the media, which varied from $2 \%$ to $10 \%$. Low serum content resulted in poor cell survival after 4 days of cultivation, whereas $5 \%$ and $10 \%$ FCS were optimal for cell proliferation and differentiation. For both the $1 \times 10^{4}$ and $5 \times 10^{4} / \mathrm{cm}^{2}$ densities, the observed cellular phenotypes were as follows: retinal ganglion celllike phenotype (4-6\% of the cells, Figure 1B), bipolar celllike phenotype (5-10\%; Figure 1C), astrocyte and Müller glia-like cells (5-8\%; Figure 1D), retinal photoreceptor-like cells (3-6\%; Figure 1E), as well as other cells with neuronal morphology, which could be considered ganglion, bipolar, horizontal and amacrine-like cells (40-50\% of the cells), and undifferentiated cells (20-27\%).

In further experiments, RPCs plated at the medium density $\left(5 \times 10^{4}\right.$ cells $\left./ \mathrm{cm}^{2}\right)$ were used throughout the study. Cultured RPCs were antibody-stained for retinal marker proteins: blue and green opsins, expressed in cones; rod marker rhodopsin; $\beta$-arrestin and recoverin from both photoreceptor cell types; calbindin, PKC $\alpha$ isoform and calretinin, which are markers for horizontal cells, bipolar cells and retinal amacrine interneurons, respectively; GFAP, characteristic of Müller glia cells, as well as for the proliferating cell marker Ki 67 and neuroepithelial stem cell marker nestin. Cell staining was analyzed both by flow cytometry (Table 1) and fluorescent microscopy. Cells were found to be immunoreactive against all of the above marker protein-specific antibodies at very different levels ranging from $3.6 \%$ of the cells for GFAP to $43.1 \%$ for green/blue opsins. These data strongly support the RPC

Table 1 Flow cytometry data on marker protein expression in RPCs

\begin{tabular}{|c|c|c|c|c|}
\hline \multirow[t]{2}{*}{ Marker protein $^{\mathrm{a}}$} & Passage $10^{\mathrm{b}}$ & Passage $10+\mathrm{RA}^{\mathrm{c}}$ & Passage $50^{\mathrm{b}}$ & Passage $50+\mathrm{RA}^{\mathrm{c}}$ \\
\hline & \multicolumn{4}{|c|}{$\%$ of expressed cells } \\
\hline GFAP & $3.6 \pm 1.1$ & $4.9 \pm 0.9$ & $2.6 \pm 0.7$ & $4.2 \pm 0.9$ \\
\hline Calbindin & $33.6 \pm 2.7$ & $36.64 \pm 2.8$ & $34.5 \pm 2.5$ & $18.4 \pm 1.4$ \\
\hline Calretinin & $26.1 \pm 1.9$ & $22.8 \pm 1.9$ & $23.6 \pm 2.0$ & $21.6 \pm 1.9$ \\
\hline$\beta$-Arrestin & $27.6 \pm 2.1$ & $14.3 \pm 1.5$ & $16.8 \pm 1.7$ & $12.3 \pm 1.2$ \\
\hline Recoverin & $36.7 \pm 2.1$ & $25.6 \pm 1.8$ & $34.8 \pm 2.2$ & $14.2 \pm 0.9$ \\
\hline Rhodopsin & $12.1 \pm 0.9$ & $23.6 \pm 1.1$ & $6.8 \pm 0.9$ & $13.1 \pm 1.4$ \\
\hline Green/ blue opsins & $30.4 \pm 2.7$ & $43.1 \pm 2.1$ & $21.8 \pm 2.3$ & $35.7 \pm 2.2$ \\
\hline Nestin & $8.1 \pm 1.2$ & $5.5 \pm 0.9$ & $12.2 \pm 1.8$ & $7.3 \pm 1.0$ \\
\hline Ki 67 & $30.3 \pm 3.0$ & $26.8 \pm 2.1$ & $55.1 \pm 2.0$ & $28.5 \pm 1.3$ \\
\hline
\end{tabular}

${ }^{a}$ Marker protein expression investigated in at least three independent experiments.

${ }^{\mathrm{b}}$ Without RA.

${ }^{\mathrm{c}}$ With RA added to the medium. 
origin of the isolated cell culture. During at least first 15 passages, the cell culture entirely retained all of the abovementioned morphological and marker protein expression patterns, as examined visually and immunocytochemically for every fifth passage of the RPC culturing. Like Ezeonu et al. [19], we have been able to passage these cells up to the 50th passage. However, by passage 20 RPCs have lost the ability to differentiate into cells with the morphology of mature retinal neuronal cells. Also, these cells did not keep their marker protein expression patterns. Therefore, multipotent RPCs from passage 10 were used for further experiments.

\section{$R A$ affects $R P C$ viability by reducing apoptosis}

We attempted to study the influence of RA on the RPC phenotype. RA was added to 5\% FCS culture media, and the resulting cell phenotypes were analyzed 7 days later both visually and by flow cytometry. In good agreement with previous data, RA-treated RPCs demonstrated an altered expression of several marker proteins [21] (Table 1). However, RA treatment did not alter RPC morphologies, and the relative ratios of photoreceptor, amacrine, bipolar, ganglion and Müller glia cell-like morphotypes remained essentially the same. Unexpectedly, RA-treated cells were characterized by an improved viability as the treatment led to $1.84 \pm 0.1$-fold higher cell yield after 7 days of culturing in a series of six independent experiments whereby viable cells were examined by the trypan blue exclusion assay. Interestingly, essentially the same RA effect (increased cell viability and altered characteristic marker protein expression profile) was observed for RPCs in passage 50 as well, when the cells were unable to develop adult retinal neuron-like shapes. These data suggest that RA affects both RPC survival and phenotype.

In order to investigate whether the enhanced RPC survival is due to reduced apoptosis or a proliferative activity of RA, we performed several assays. For cell growth and proliferation studies, both colorimetric MTT and flow cytometric BrdU assays were performed. A PI analysis revealed that RPC cell cycle progression was not affected by RA treatment. Both MTT- and BrdU-based proliferation assays showed that RA had no effect on RPC proliferation as well (Figure 2). RA effects on RPC proliferation were monitored 24-h after the addition of RA using the MTT reagent and 7 days after by BrdU staining. The main message of Figure 2A is that RA has no initial toxic effect on RPCs in a wide range of concentrations. For a short period of incubation with RA like $24 \mathrm{~h}$, no significant RA effect on RPC viability is visible. However, for a period of 7 days this effect was significant (1.7-1.9 greater cell yield when RA was added, see above), whereas the proportion of proliferating cells remained essentially the same (Figure 2B), thus suggesting
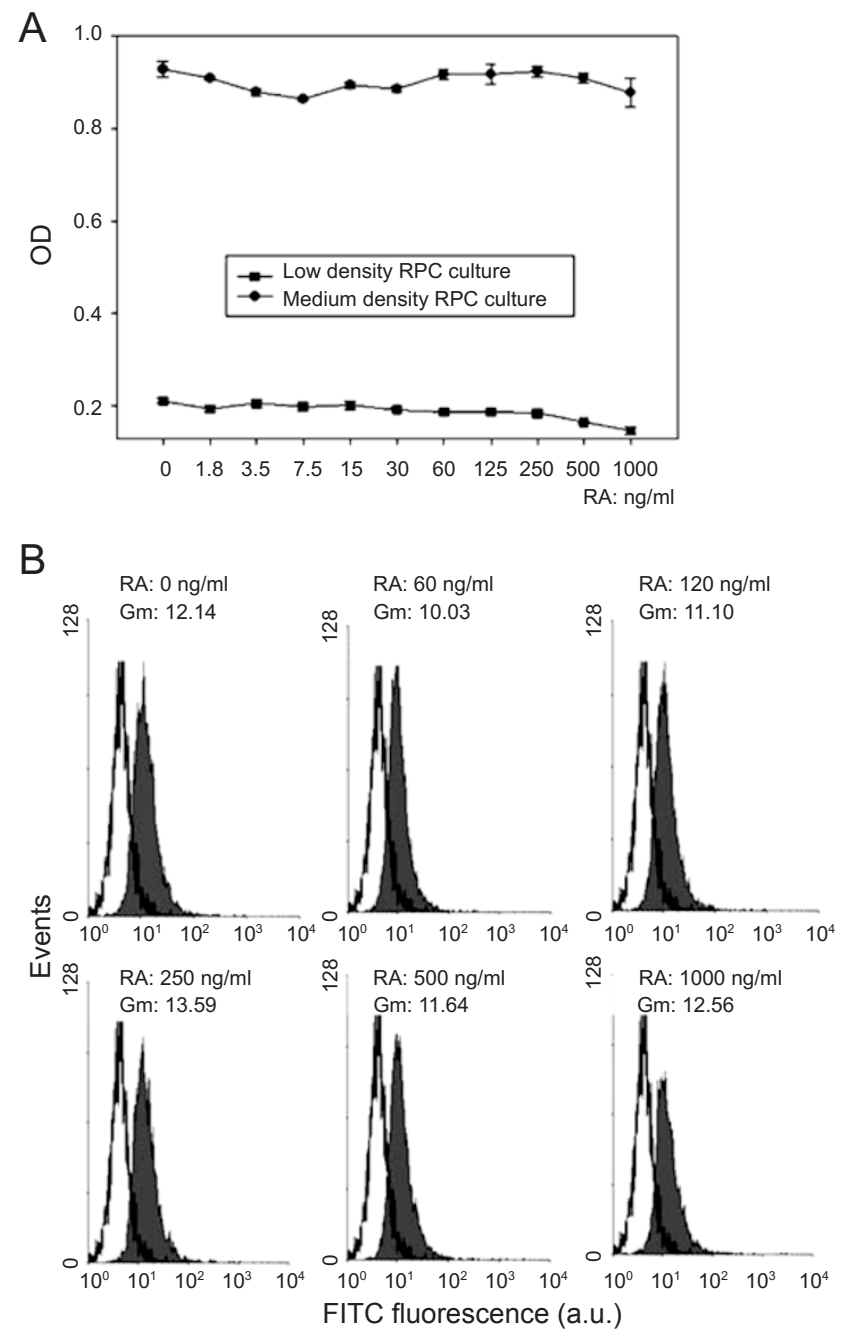

Figure 2 RA effect on RPC proliferation. (A) MTT assay. Low and medium density RPC cultures $\left(1 \times 10^{4}\right.$ and $5 \times 10^{4}$ cells $/ \mathrm{cm}^{2}$, respectively) exposed to different RA concentrations ranging from 0 to 1000 $\mathrm{ng} / \mathrm{ml}$ were incubated with the MTT reagent. The cells were washed, solubilized with $10 \%$ SDS, and the OD was measured at $570 \mathrm{~nm}$. (B) BrdU assay. Flow cytometry analyses of BrdU incorporation in RPCs exposed to different concentrations of RA, using FITC fluorescence (a.u.). Left peaks (transparent) - control fluorescence (BrdU-free culture medium); right peaks (dense) $-10 \mu \mathrm{M}$ BrdU added to the medium. RA concentrations and fluorescence peak positions $(\mathrm{Gm})$ are indicated.

that the increased RPC viability mediated by RA is not due to an enhanced RPC proliferation. It should be mentioned here that the proliferative marker Ki 67 expression was observed at essentially the same level for both RA-treated and untreated RPCs from passage $10(26.8 \pm 2.1 \%$ and $30.3 \pm 3.0 \%$ cells, respectively) and for RA-treated RPCs from passage $50(28.5 \pm 1.3 \%$ cells). In our experiments, 
untreated RPCs from passage 50 demonstrated $\sim$ twofold higher expression of Ki 67 (55.1 $\pm 2.0 \%$ cells) (Table 1). However, this increased expression of Ki 67 did not correlate with increased proliferation rate, as measured in BrdU staining experiments; instead, the proliferation rate was shown to be the same for both RA-treated and untreated RPCs from both passages 10 and 50. Although $\mathrm{Ki} 67$ is a widely used marker, it is known that the data on the expression of different proliferative marker proteins, such as Ki 67, PCNA or Mcm-2, do not always accurately reflect cell proliferation status, and that other, more direct approaches like BrdU staining are preferred for counting proliferating cells. Moreover, the biological function of Ki 67 remains unclear. Therefore, the enhanced Ki 67 expression by the passage 50 RPCs might be unrelated to cell division.

To monitor the effect of RA on apoptosis in RPCs, we used the PI-based screening system. The data (Table 2) demonstrated that for RPCs from passages 10 and 50, plated at both medium and high densities, addition of RA to the culture media led to $\sim 23 \%$ decrease in the number of apoptotic cells. To confirm the PI screening results, we employed the Annexin V assay. Importantly, a similar decrease (by $\sim 23 \%$ ) in the number of apoptotic cells was observed for RA-treated cells from both passage 10 and passage 50 using this approach (Figure 3 ), thus demonstrating the reliability of the PI-based system for apoptosis

Table 2 Influence of different factors on apoptosis in RPCs

\begin{tabular}{|c|c|c|c|}
\hline Factors & Apoptotic cells $(\%)^{1}$ & Effect $(\%)^{2}$ & Comments $^{3}$ \\
\hline- & $23.1 \pm 0.9$ & - & Controls \\
\hline RA & $17.7 \pm 0.9$ & -23.4 & Retinoic acid added (+RA) \\
\hline RA & $25.2 \pm 1.2$ & -22.7 & High cell density + RA \\
\hline- & $20.7 \pm 0.8$ & - & RPCs at passage 50 \\
\hline RA & $22.5 \pm 0.7$ & -23.7 & RPCs at passage 50 , high cell density + RA \\
\hline$\beta$-Amyloid & $44.5 \pm 1.4$ & +92.6 & $\beta$-Amyloid \\
\hline$\beta$-Amyloid + RA & $42.2 \pm 1.7$ & +82.7 & $\beta$-Amyloid + RA \\
\hline C16-Cer & $26.0 \pm 0.7$ & +12.6 & Ceramide C16 \\
\hline GM1 & $26.2 \pm 0.6$ & +13.4 & Ganglioside GM1 \\
\hline $\mathrm{GM} 1+\mathrm{RA}$ & $26.6 \pm 0.9$ & +15.2 & Ganglioside GM1 + RA \\
\hline TNF $\alpha$ & $23.8 \pm 1.1$ & +3.0 & Tumor necrosis factor $\alpha$ \\
\hline TNF $\alpha+$ RA & $22.9 \pm 1.0$ & -0.9 & Tumor necrosis factor $\alpha+\mathrm{RA}$ \\
\hline H89 & $27.9 \pm 1.1$ & +20.9 & PKA inhibitor \\
\hline $\mathrm{H} 89+\mathrm{RA}$ & $30.5 \pm 0.9$ & +31.9 & PKA inhibitor + RA \\
\hline 8-Br-cAMP & $19.9 \pm 0.7$ & -13.9 & PKA activator \\
\hline 8-Br-cAMP + RA & $17.6 \pm 0.3$ & -23.9 & PKA activator $+\mathrm{RA}$ \\
\hline HBDDE & $16.0 \pm 0.8$ & -30.5 & PKC inhibitor \\
\hline
\end{tabular}

${ }^{1}$ Percentage of apoptotic RPCs found with the PI assay from at least three independent experiments.

${ }^{2}$ Effect on RPC apoptosis, percentage relative to the corresponding controls.

${ }^{3}$ When not specified, RPCs from passage 10 plated at a density of $5 \times 10^{4}$ cells $/ \mathrm{cm}^{2}$. 
A

RA-

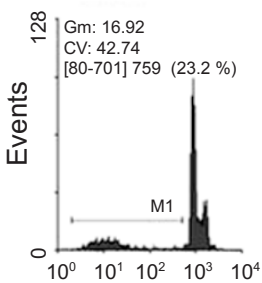

RA-

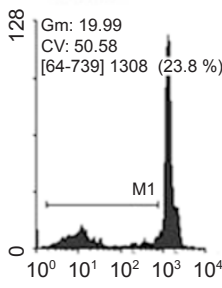

Medium density retinal progenitor cells (RPCs) Apoptotic cells (\%) 23.2

23.8

RA-

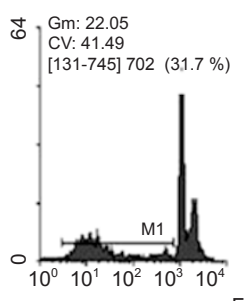

FL3LOG

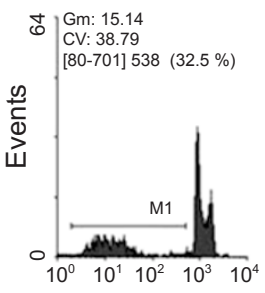

Medium density retinal progenitor cells (RPCs)
RA-

medium density
RA+

$\stackrel{\infty}{\sim}, \mathrm{Gm}: 13.86$

CV: 42.08

[80-701] $539(25.2 \%)$

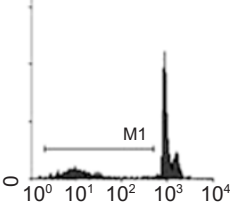

O
$\stackrel{0}{N}$ Gm: 16.68

CV: 39.79

[80-701] $575(17.8 \%)$

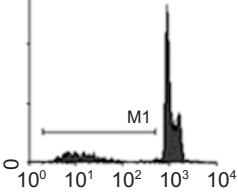

17.8

25.2
RA+

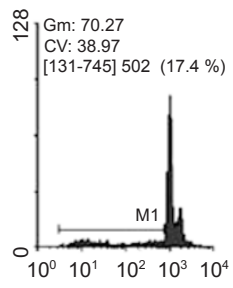

17.4

RA+

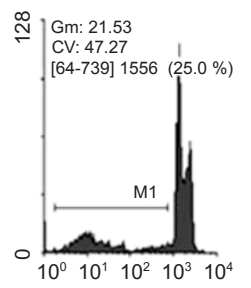

25.0

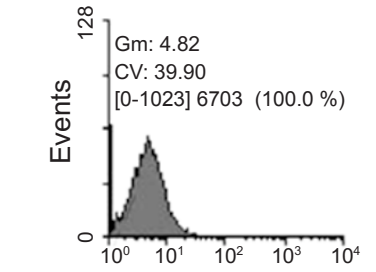

RA-

high density

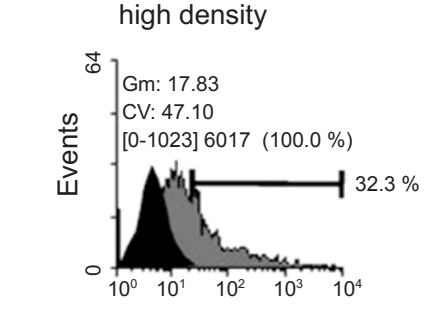

B
¿

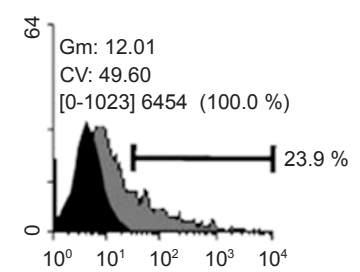

RA+

high density

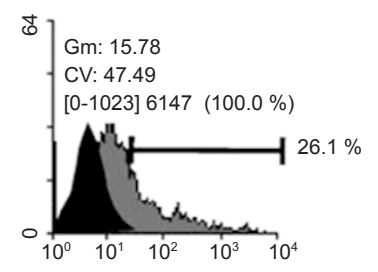

$\mathrm{RA}+$

medium density
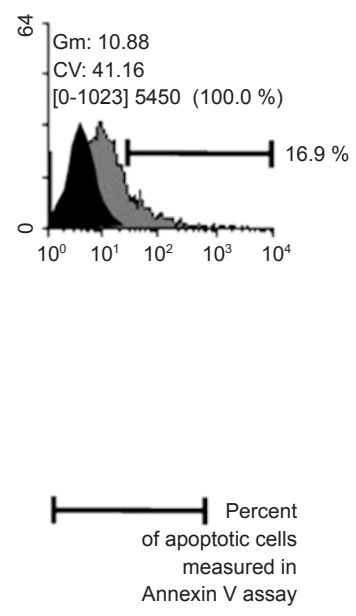

Figure 3 Long-term effect of RA on RPC apoptosis. RPCs were harvested after 7 days of RA treatment and analyzed with either PI or Annexin V staining assays. Both assays produce a consistent result of $\sim 23 \%$ decrease in apoptotic cells when RA is added to the culture media for both medium- and high-density plated RPCs. (A) Representative results of the PI assay. Medium- and high-density RPC cultures $\left(5 \times 10^{4}\right.$ and $5 \times 10^{5}$ cells $\left./ \mathrm{cm}^{2}\right)$ were grown in RA-containing or in RA-free medium. Apoptotic cells were measured in a PI-based flow-cytometer assay. Each representative histogram is shown in duplicate. (B) Representative histograms of the Annexin V assay. Medium- and high-density RPCs were grown in RA-containing or in RA-free medium. Apoptotic cells were directly stained with Annexin V-FITC conjugate and measured on a flow cytometer. Control experiment: attached RPCs stained after 24-h culturing in RA-free medium with $10 \%$ FCS. 
measuring in this study. This decrease of $\sim 23 \%$ seems reasonable to explain the approximately twofold higher cell yield in RA-treated RPC culture after 7 days. Indeed, even $\sim 10 \%$ decrease in apoptotic human HL-60 cells provided significantly higher augmentation of the cell yield [23]. We, therefore, conclude that the observed effect of RA on RPC viability is exclusively due to the RA-mediated antiapoptotic activity in these cells.

PKA and PKC antagonistic activities are probable mediators for the RA-induced anti-apoptotic effect

We further tried to explore the mechanisms underlying the anti-apoptotic effect of RA in RPCs. Many apoptotic pathways known to be activated by specific proteins or chemicals operate in neuronal cells, although the specific details may differ depending on the tissue origin. RA interference with any of such pathways would provide a hint for understanding of the mechanism of its anti-apoptotic activity. To assess RA effects, the following known apoptosis inducers were used: $\beta$-amyloid, ceramide $\mathrm{C} 16$, anti-Fas antibodies (serve as in vitro functional analogs of Fas ligand), TNF- $\alpha$ and GM1 ganglioside. Of these, only $\beta$-amyloid, ceramide $\mathrm{C} 16$ and ganglioside GM1 were able to induce apoptosis in RPCs as manifested by a $92 \%, 13 \%$ and $13 \%$ increase in the apoptotic cell number, respectively, compared with controls. However, RA apparently did not interfere with any of the apoptotic inducers mentioned above (Table 2), thus suggesting that its effect might involve other signaling pathway(s). As bcl-2 and P53 proteins are among the most important players in signaling cascades responsible for regulating cell death and survival, we further antibody-stained RA-treated and untreated RPCs for these proteins. Somewhat unexpectedly, the expression of these markers was unaffected by the addition of RA to the culture medium (Table 1).

However, as revealed by a previous marker protein expression assay, expression of the PKC $\alpha$ isoform was upregulated in RA-treated cells (Table 1). PKC $\alpha$ is known to be not only a bipolar cell marker, but also one of the key $\mathrm{Ser} / \mathrm{Thr}$ protein kinases involved in multiple signal-transduction pathways. RT-PCR analyses with primers specific to nine different $\mathrm{PKC}$ isoforms revealed that the transcription of four of them (PKC $\alpha, \varepsilon, \zeta \zeta$ and $\lambda / \mathrm{l}$ ) was $\sim$ four- to eightfold upregulated in RA-treated cells (Figure 4A), with the PKC $\zeta$ transcript level being the highest. The other PKC isoforms were expressed at similar levels in both treated and untreated cells. To address the question as to whether the effect of RA is due to an increased PKC activity, we performed assays with two known PKC inhibitors, HBDDE and BIM, and with the specific PKC activator PMA. Surprisingly, PKC activation resulted in increased apoptosis $(+12 \%$ as compared with controls), whereas PKC inhibition with both
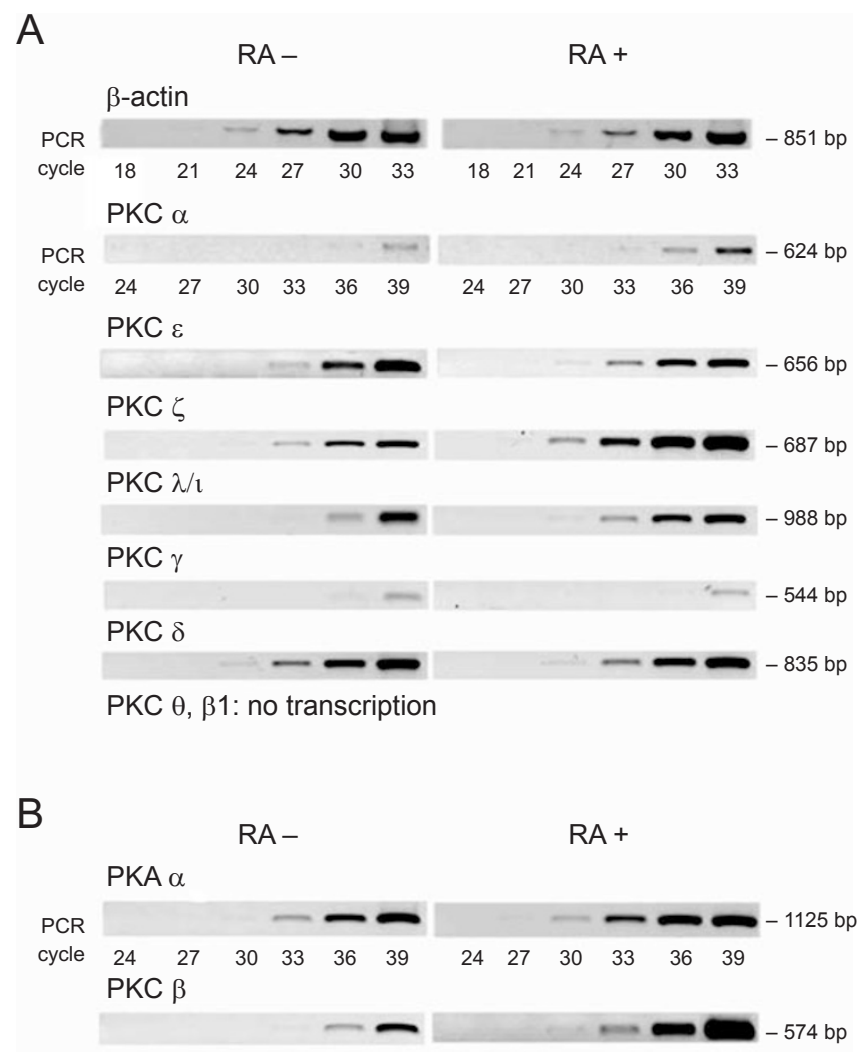

Figure 4 Representative results of RT-PCR analyses of cDNAs obtained from RA-treated (RA+) and untreated (RA-) RPCs. All RT-PCR experiments were reproduced at least three times using independent cDNA preparations. (A) RT-PCR amplification with primers specific to $\beta$-actin (control amplification serving as an internal standard) and to different PKC isoforms: PKC $\alpha, \varepsilon, \zeta, \lambda / \imath, \gamma, \delta, \theta$ and $\beta 1$. (B) RT-PCR amplification with primers specific to PKA catalytic $\alpha$ and regulatory $\beta$ subunits. PCR product lengths are indicated.

HBDDE and BIM decreased the proportion of apoptotic cells $(-31 \%$ and $-21 \%$, respectively; Table 2$)$. Thus, RAinduced PKC activation cannot explain the anti-apoptotic effect of RA on RPCs. We hypothesized that the effect of RA might be mediated by another protein kinase, known as cAMP-dependent Ser/Thr protein kinase, or PKA. It is known from the literatures that in many cases both PKC and PKA are activated by RA [24]; more interestingly, they frequently act as functional antagonists of each other [25]. To test this hypothesis, we performed RT-PCR experiments with primers specific to two PKA subunits: catalytic $\alpha$ and regulatory $\beta$. RT-PCRs revealed that the expression of both PKA subunits was $~$ eight-fold upregulated in RA-treated cells, thus suggesting PKA activation by RA (Figure 4B). This hypothesis was further supported by experiments with the PKA inhibitor H89 and activator 8-Br-cAMP (Table 
2). PKA activation resulted in reduced apoptosis $(-14 \%)$, whereas PKA inhibition increased RPC apoptosis $(+21 \%)$. Interestingly, when PKA activator and RA were added together to RPCs, the effect on RPC apoptosis was almost identical to that caused by RA alone, consistent with the notion that PKA acts in the RA-mediated pathway. We, therefore, conclude that RA stimulation of RPCs increases the expression of both PKA and PKC, which may act as functional antagonists by decreasing and increasing apoptosis, respectively. Such double kinase activation might be advantageous for tight regulation of this important process by providing an instrument for a quick and balanced response to new external or intracellular signals.

\section{Discussion}

Our study suggests RA-mediated activation of two protein kinases (PKA and PKC) acting as functional an-

A

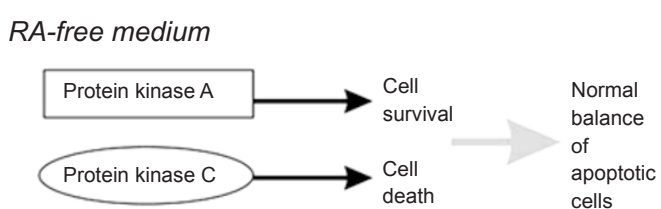

B

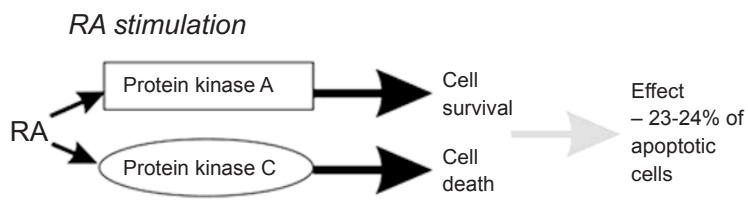

C

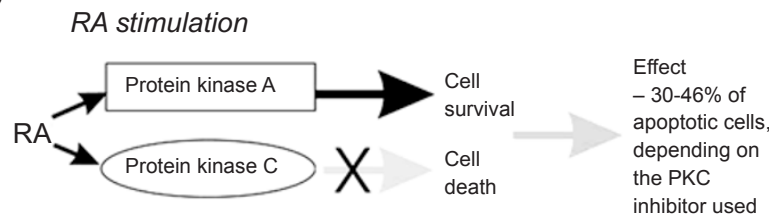

D

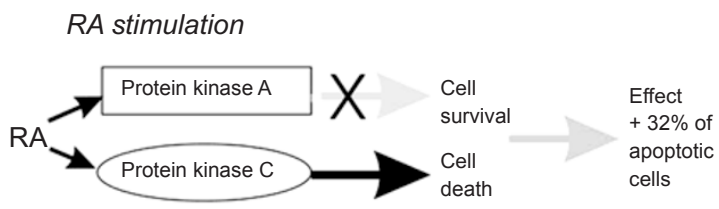

Figure $5 \mathrm{~A}$ model explaining the effects of RA and protein kinase inhibitors on RPC survival. Full data sets are presented in Table 2. (A) No RA or protein kinase inhibitors added to RPCs. (B) RA added to the culture medium. (C) RA and PKC inhibitors (BIM or HBDDE) added to the medium. (D) RA and PKA inhibitor (H89) added to the medium. tagonists. The PKA/PKC inhibitor-based analyses provide another line of evidence for such a hypothesis (Figure 5A). According to the model, RA stimulation activates both PKA and PKC, with the former supporting RPC survival, whereas the latter increasing apoptosis (Figure 5B). This model predicts that RA stimulation of RPCs accompanied by treatment with PKC inhibitors should result in a greater anti-apoptotic activity than that induced by RA alone. In this case PKA will be stimulated, while the PKC-mediated compensatory mechanism, normally suppressing the PKA effect, will be inhibited (Figure 5C). This prediction was tested by an inhibitor assay: treatment with RA and the PKC inhibitor indeed resulted in a significantly better antiapoptotic effect $(-46 \%$ and $-30 \%$ for HBDDE and BIM, respectively) than that of RA alone $(-23 \%)$. In contrast, PKA inhibition together with RA treatment would cause PKC activation that is not balanced by PKA (Figure 5D), and therefore would lead to increased apoptosis compared with cells treated with RA alone or untreated cells. This prediction was also confirmed by experimental data $(+32 \%$ vs $-23 \%$ and $0 \%$, respectively).

It was previously reported that PKA and PKC may serve as antagonists for each other in RA-treated cells, which is in good agreement with the present findings. For instance, in Sertoli cells from rat testis RA induced differentiation, with the effect of RA being enhanced by PKC activation and reduced by upregulation of PKA [24]. In HepG2 cells, both RA treatment and PKA activation resulted in upregulation of the cholesterol seven $\alpha$-hydroxylase gene transcription, whereas PKC activation led to silencing of this gene [25]. In human neuroblastoma, PKC activation mediated an anti-apoptotic effect via Bcl-2 activation, whereas PKA suppressed Bcl-2 activation and increased the proportion of apoptotic cells [26]. An overwhelming majority of RAinduced effects in neuronal cells, as described in the literature, have been ascribed to cell maturation, differentiation, control of proliferation or to cell death, with very few published examples of RA-mediated anti-apoptotic effects. For retinal cells, only indirect evidence was obtained recently: Zhou et al. [27] reported that inhibition of RA receptors in the developing mouse eye stimulated apoptosis in neonatal mice retinas. In this paper, we propose a mechanism for the anti-apoptotic activity of RA in RPCs, which comprises activation of PKA and PKC. Interestingly, structural data support the idea that several PKC isoforms, normally activated by increased concentrations of diacylglycerol and calcium ions, could also be directly activated by RA, as they contain potential binding sites for two RA molecules [28]. An interesting model for PKC and PKA double activation (although not RA-dependent) was previously suggested for B lymphocytes: high intracellular calcium concentrations via calmodulin lead to increased cAMP concentration, 
which modulates PKA activation. Simultaneously, calcium ions activate PKC isoforms $\alpha, \delta$ and $\varepsilon$ (in our experiments, transcription of $\alpha, \varepsilon, \zeta$ and $\lambda / \mathrm{\imath}$ isoforms was activated by RA). The mechanism proposed in our model (Figure 5) relies mostly on the inhibitor/activator studies performed with well-known PKA and PKC inhibitors and activators: H89 [29], 8-Br-cAMP [30], HBDDE [31], BIM [32] and PMA [33]. A more detailed analysis of RA-mediated antiapoptotic mechanisms is a subject for future studies. The results reported here might be of considerable usefulness in creating better in vitro RPC systems with increased cell viability for modeling eye development and/or for future stem cell-based strategies to treat retinopathy.

\section{Acknowledgments}

This work was supported by the Molecular and Cellular Biology Program of the Presidium of the Russian Academy of Sciences, by the Grant MK-2833.2004.4 of the President of the Russian Federation and by an anonymous sponsor. We are grateful to Dr Boris Glotov for valuable discussion and comments to the manuscript and to Professor Eugene D Sverdlov for overall support of this research.

\section{References}

1 Marquardt T, Gruss P. Generating neuronal diversity in the retina: one for nearly all. Trends Neurosci 2002; 25:32-38.

2 Goldman S. Stem and progenitor cell-based therapy of the human central nervous system. Nat Biotechnol 2005; 23:862-871.

3 Tropepe V, Coles BL, Chiasson BJ, et al. Retinal stem cells in the adult mammalian eye. Science 2000; 287:2032-2036.

4 Coles BL, Horsford DJ, McInnes RR, van der Kooy D. Loss of retinal progenitor cells leads to an increase in the retinal stem cell population in vivo. Eur J Neurosci 2006; 23:75-82.

5 Li S, Mo Z, Yang X, Price SM, Shen MM, Xiang M. Foxn4 controls the genesis of amacrine and horizontal cells by retinal progenitors. Neuron 2004; 43:795-807.

6 Shi Y, Chichung Lie D, Taupin P, et al. Expression and function of orphan nuclear receptor TLX in adult neural stem cells. Nature 2004; 427:78-83.

7 McBride JL, Behrstock SP, Chen EY, et al. Human neural stem cell transplants improve motor function in a rat model of Huntington's disease. J Comp Neurol 2004; 475:211-219.

8 Jakel RJ, Schneider BL, Svendsen CN. Using human neural stem cells to model neurological disease. Nat Rev Genet 2004; 5:136144.

9 Sugaya K. Neuroreplacement therapy and stem cell biology under disease conditions. Cell Mol Life Sci 2003; 60:1891-1902.

10 Jones BW, Watt CB, Frederick JM, et al. Retinal remodeling triggered by photoreceptor degenerations. J Comp Neurol 2003; 464:1-16.

11 Lund RD, Adamson P, Sauve Y, et al. Subretinal transplantation of genetically modified human cell lines attenuates loss of visual function in dystrophic rats. Proc Natl Acad Sci USA 2001; 98:9942-9947.
12 Meyer JS, Katz ML, Maruniak JA, Kirk MD. Embryonic stem cell-derived neural progenitors incorporate into degenerating retina and enhance survival of host photoreceptors. Stem Cells 2006; 24:274-283.

13 Wegewitz U, Gohring I, Spranger J. Novel approaches in the treatment of angiogenic eye disease. Curr Pharm Des 2005; 11:2311-2330.

14 Sakaguchi DS, Van Hoffelen SJ, Young MJ. Differentiation and morphological integration of neural progenitor cells transplanted into the developing mammalian eye. Ann NY Acad Sci 2003; 995:127-139.

15 Sheedlo HJ, Turner JE. Influence of a retinal pigment epithelial cell factor(s) on rat retinal progenitor cells. Brain Res Dev Brain Res 1996; 93:88-99.

16 Yang P, Seiler MJ, Aramant RB, Whittemore SR. In vitro isolation and expansion of human retinal progenitor cells. Exp Neurol 2002; 177:326-331.

17 Klassen H, Ziaeian B, Kirov, II, Young MJ, Schwartz PH. Isolation of retinal progenitor cells from post-mortem human tissue and comparison with autologous brain progenitors. J Neurosci Res 2004; 77:334-343.

18 Bavister BD, Wolf DP, Brenner CA. Challenges of primate embryonic stem cell research. Cloning Stem Cells 2005; 7:82-94.

19 Ezeonu I, Wang M, Kumar R, Dutt K. Density-dependent differentiation in nontransformed human retinal progenitor cells in response to basic fibroblast growth factor- and transforming growth factor-alpha. DNA Cell Biol 2003; 22:607-620.

20 Kelley MW, Turner JK, Reh TA. Retinoic acid promotes differentiation of photoreceptors in vitro. Development 1994; 120:20912102.

21 Levine EM, Fuhrmann S, Reh TA. Soluble factors and the development of rod photoreceptors. Cell Mol Life Sci 2000; 57:224234.

22 Gratzner HG. Monoclonal antibody to 5-bromo- and 5-iododeoxyuridine: a new reagent for detection of DNA replication. Science 1982; 218:474-475.

23 Kondo T, Matsuda T, Kitano T, et al. Role of c-jun expression increased by heat shock- and ceramide-activated caspase- 3 in HL-60 cell apoptosis. Possible involvement of ceramide in heat shock-induced apoptosis. J Biol Chem 2000; 275:7668-7676.

24 Braun KW, Vo MN, Kim KH. Positive regulation of retinoic acid receptor alpha by protein kinase $\mathrm{C}$ and mitogen-activated protein kinase in sertoli cells. Biol Reprod 2002; 67:29-37.

25 Crestani M, Stroup D, Chiang JY. Hormonal regulation of the cholesterol 7 alpha-hydroxylase gene (CYP7). J Lipid Res 1995; 36:2419-2432.

26 Itano Y, Ito A, Uehara T, Nomura Y. Regulation of Bcl-2 protein expression in human neuroblastoma SH-SY5Y cells: positive and negative effects of protein kinases $\mathrm{C}$ and $\mathrm{A}$, respectively. $\mathrm{J}$ Neurochem 1996; 67:131-137.

27 Zhou J, Kochhar DM. Regulation of AP-2 and apoptosis in developing eye in a vitamin A-deficiency model. Birth Defects Res A Clin Mol Teratol 2003; 67:41-53.

28 Radominska-Pandya A, Chen G, Czernik PJ, et al. Direct interaction of all-trans-retinoic acid with protein kinase C (PKC). Implications for PKC signaling and cancer therapy. J Biol Chem 2000; 275:22324-22330.

29 Chijiwa T, Mishima A, Hagiwara M, et al. Inhibition of forskolininduced neurite outgrowth and protein phosphorylation by a newly 
synthesized selective inhibitor of cyclic AMP-dependent protein kinase, $N$-[2-( $p$-bromocinnamylamino)ethyl]-5-isoquinolinesulfonamide $(\mathrm{H}-89)$, of PC12D pheochromocytoma cells. J Biol Chem 1990; 265:5267-5272.

30 Chen TC, Hinton DR, Zidovetzki R, Hofman FM. Up-regulation of the cAMP/PKA pathway inhibits proliferation, induces differentiation, and leads to apoptosis in malignant gliomas. Lab Invest 1998; 78:165-174.

31 Ma R, Kudlacek PE, Sansom SC. Protein kinase Calpha participates in activation of store-operated $\mathrm{Ca} 2+$ channels in human glomerular mesangial cells. Am J Physiol Cell Physiol 2002; 283:C1390-C1398

32 Coultrap SJ, Sun H, Tenner TE Jr., Machu TK. Competitive antagonism of the mouse 5-hydroxytryptamine 3 receptor by bisindolylmaleimide I, a "selective" protein kinase C inhibitor. J Pharmacol Exp Ther 1999; 290:76-82.

33 Mizuguchi J, Ji YY, Nakabayaschi H, et al. Protein kinase C activation blocks anti-IgM-mediated signaling BAL17 B lymphoma cells. J Immunol 1987; 139:1054-1059. 